\title{
Editorial
}

\section{Function Spaces, Hyperspaces, and Asymmetric and Fuzzy Structures}

\author{
Salvador Romaguera, ${ }^{1}$ Gerald Beer, ${ }^{2}$ and Manuel Sanchis ${ }^{3}$ \\ ${ }^{1}$ Instituto Universitario de Matemática Pura y Aplicada, Universitat Politècnica de València, 46022 Valencia, Spain \\ ${ }^{2}$ Department of Mathematics, California State University, Los Angeles, CA 90032, USA \\ ${ }^{3}$ Institut de Matemàtiques i Aplicacions de Castelló (IMAC), Universitat Jaume I, Campus del Riu Sec S/N, 12071 Castelló, Spain
}

Correspondence should be addressed to Salvador Romaguera; sromague@mat.upv.es

Received 2 April 2013; Accepted 2 April 2013

Copyright (c) 2013 Salvador Romaguera et al. This is an open access article distributed under the Creative Commons Attribution License, which permits unrestricted use, distribution, and reproduction in any medium, provided the original work is properly cited.

Not long after L. A. Zadeh proposed in the sixties his theory of fuzzy sets, this subject became a productive field of research: fuzzy structures not only generalize most of the familiar structures but also they allow obtaining interesting applications in engineering. Hyperspaces, asymmetric topology and fuzzy structures are interdisciplinary topics of increasing interest in the development of the general topology, and its applications in other areas of Mathematics and computer science, such as convex analysis and optimization, fractals and dynamical systems, mathematical economics, and image computing.

The interplay between fuzzy structures and the theory of hyperspaces and the asymmetric topology is a topic of broad appeal, encouraging new research on such topics and in many cases defining emerging research directions and laying the foundation for future innovations. Many of the impactful and highly cited papers in general topology and its applications have been published in the realm of this relationship.

This special issue addresses work at the interface between function spaces, hyperspaces, fuzzy structures, and asymmetric topology. Its core can be summarized as follows.

The paper "A weak comparison principle for reactiondiffusion systems" deals with certain differential equations for which uniqueness of the Cauchy problem fails. More exactly, a weak comparison principle for a reaction-diffusion system in which the nonlinear term satisfies suitable dissipative and growth conditions, ensuring existence of solutions but not uniqueness, is established. Antecedents of this research are contained in two papers by J. Valero and A. Kapustyan, published in Journal of Mathematical Analysis and Applications (vol. 323 (2006), pp. 614-633, and vol. 357 (2009), pp. 254-272), where the authors show the existence of a global attractor for this type of reaction-diffusion systems. Applications to the problem of obtaining solutions for LotkaVolterra system with diffusion, for a model of fractionalorder chemical autocatalysis with decay, and for a generalized logistic equation are given.

In the paper q-hyperconvexity in quasipseudometric spaces and fixed point theorems the authors extend several properties of the theory of hyperconvexity in metric spaces to the asymmetric framework. Among other results, they prove the following quasimetric version of the well-known fixed point theorem of R. C. Sine and P. M. Soardi. If $(X, d)$ is a bounded $q$-hyperconvex $T_{0}$-quasimetric space and if $T$ is a nonexpansive self-map of $(X, d)$, then the fixed point set Fix $(T)$ of $T$ in $(X, d)$ is nonempty and $q$-hyperconvex. Chains of $q$-hyperconvex subspaces and the approximation of fixed points are also discussed.

The paper "On the fuzzy number space with the level convergence topology" is devoted to the study of some uniform and topological properties of the space $\mathbb{E}^{1}$ of fuzzy numbers endowed with the level convergence topology $\tau_{\ell}$. In particular the authors describe the completion $\left(\widehat{\mathbb{E}^{1}}, \widehat{\mathscr{U}}\right)$ of $\mathbb{E}^{1}$ respect to its natural uniformity, that is, the pointwise uniformity $\mathcal{U}$. Among other results, a characterization of compact subsets of $\left(\mathbb{E}^{1}, \tau_{\ell}\right)$ is presented and it is shown that the space $\left(\mathbb{E}^{1}, \tau_{\ell}\right)$ 
is a separable Baire space. Also an Arzela-Ascoli theorem is obtained for the space of $\left(\mathbb{E}^{1}, \tau_{\ell}\right)$-valued continuous functions on a locally compact topological space equipped with the compact-open topology.

In the paper "Convexity invariance of fuzzy sets under the extension principles," Zadeh's (multivariable) extension principle is applied to convexity invariance of fuzzy sets. One of the main results states that if $E$ is a real linear topological space and $\star$ is a $t$-norm, then a function $f$ from $E$ into a totally ordered set equipped with the order topology is an inverse $\star$-convex transformation if and only if the restriction of $f$ to every line segment is monotonic. Some applications of the main results to the hyperstructure convexity invariance of type-2 fuzzy sets under hyperalgebra operations and to the convexity invariance of fuzzy numbers under basic arithmetic operations are obtained. For instance, given a continuous $t$-norm, say $\star$, if $A$ and $B$ are two $\star$-convex type-2 fuzzy sets, then the generalized union $A \cup_{\star} B$ and the generalized intersection $A \cap_{\star} B$ are $\star$-convex type-2 fuzzy sets.

A notion of $\lambda$-pointwise convergence and also of $\lambda$ statistical uniform convergence in an intuitionistic fuzzy normed spaces is introduced in the paper " $\lambda$-Statistical convergence of sequences of functions in intuitionistic fuzzy normed spaces." Some properties of these concepts are proved. For example, it is showed that if a sequence $\left\{f_{k}\right\}_{k \geq 1}$ of functions from an intuitionistic fuzzy normed space $(X, \mu, \nu, \star, \diamond)$ to another intuitionistic fuzzy normed space $\left(Y, \mu^{\prime}, \nu^{\prime}, \star, \diamond\right)$ is pointwise intuitionistic fuzzy convergent on $X$ to a function $f$ with respect to $(\mu, \nu)$, then $\left\{f_{k}\right\}_{k \geq 1}$ is pointwise $\lambda$-statistical convergent with respect to the intuitionistic fuzzy norm $(\mu, v)$.

In 2000 S. Park extended the notion of $w$-distance to the quasimetric framework in order to generalize and unify different versions of Ekeland's variational principle. Park's approach was continued by several authors in recent years and generalizations of Caristi-Kirk's fixed point theorem and Takahashi type minimization theorem, among others, were generalized and improved. Motivated by the fact that, with this approach, the topology induced by a quasimetric $d$ that is also a $w$-distance for $d$ is metrizable, in the paper "Generalized contractive set-valued maps on complete preordered quasimetric spaces" the notion of $w$-distance is modified, by means of a preorder condition, to include in this theory several distinguished examples of quasimetric spaces, and some fixed point results for multivalued maps that extend well-known theorems to this setting are obtained.

In 1934 G. Birkoff showed that there exists a topology on the full self-homeomorphism group $H(X)$ of a compact metric space $X$ which makes it into a topological group and as a subspace of the Hilbert cube. In 1946 Arens relaxes the condition of compactness to local compactness by studying topologies on the group $H(X)$ which make it a topological group and, at the same time, provide continuity of the evaluation function, that is, the so-called admissible group topologies. From this starting point, the paper "Topologizing hemoemomorphism groups" presents a survey of results relative to a special kind of function spaces like the full group of homeomorphisms of a Tychonoff space and its subgroups.

Salvador Romaguera Gerald Beer Manuel Sanchis 


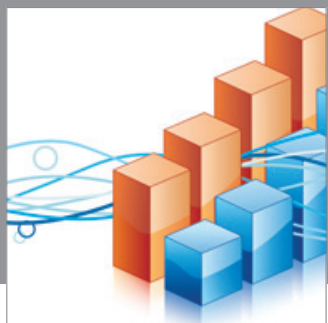

Advances in

Operations Research

mansans

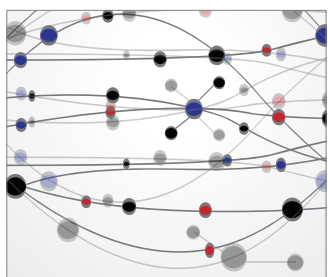

The Scientific World Journal
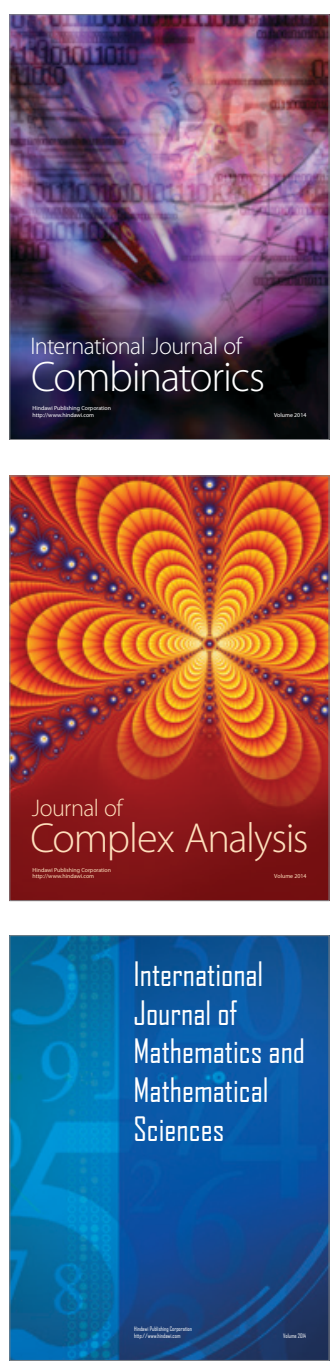
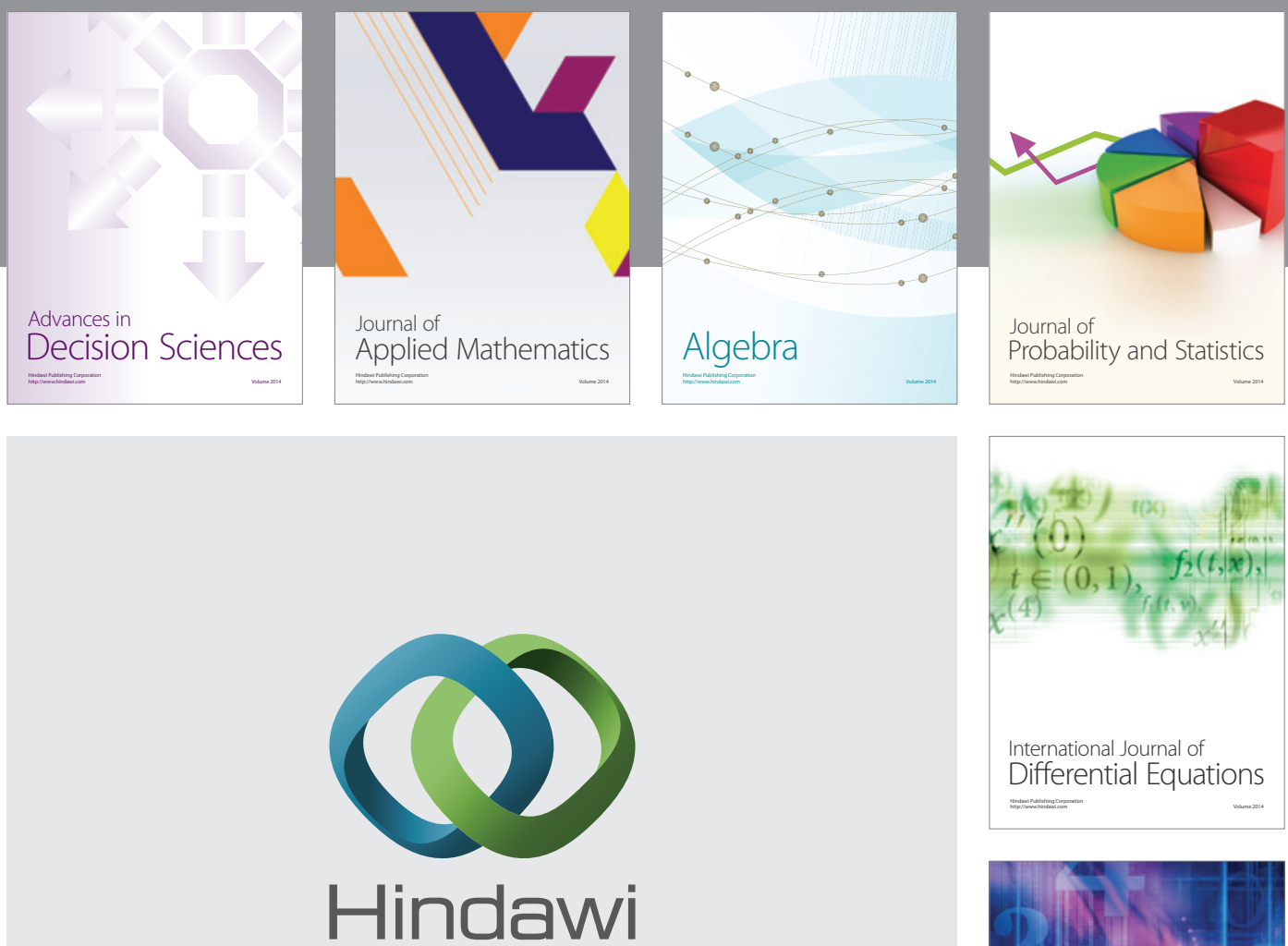

Submit your manuscripts at http://www.hindawi.com
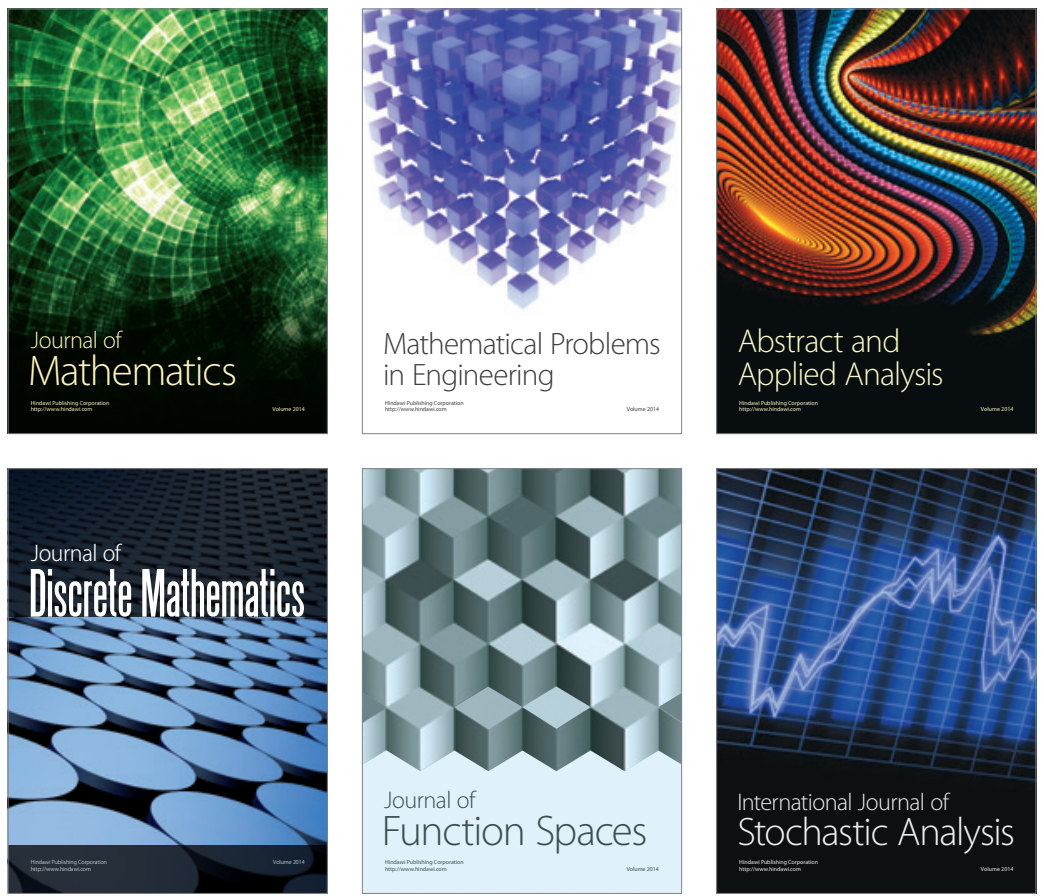

Journal of

Function Spaces

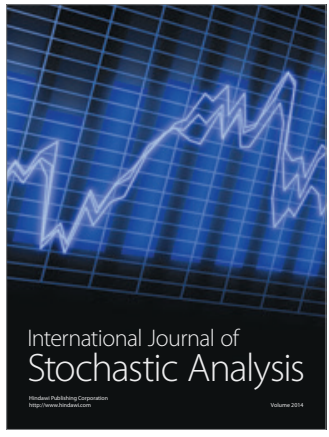

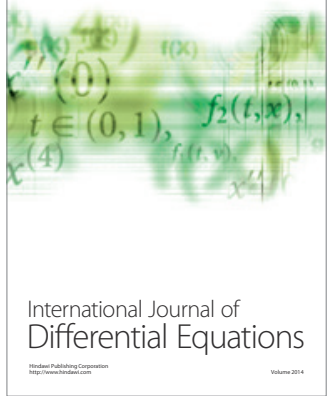
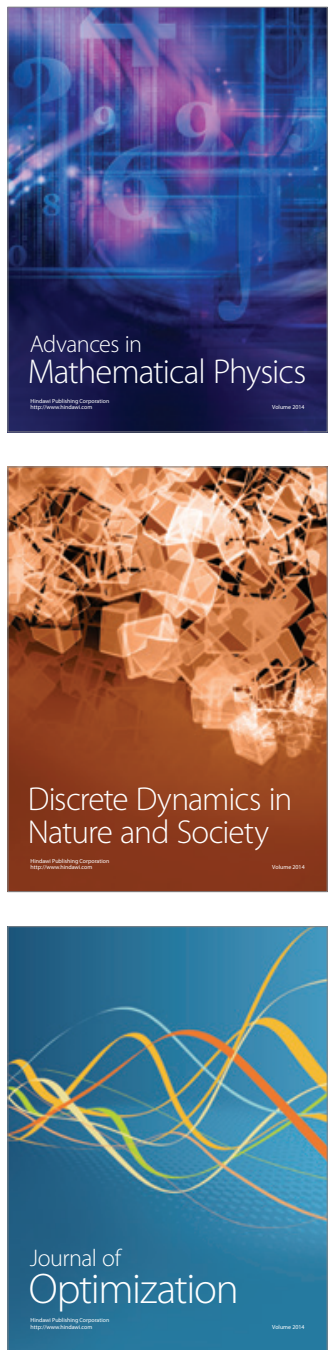